\title{
Estructura cariotípica de dos poblaciones de Rhamdia quelen (Pisces, Siluriformes) de los ríos Paraná y Paraguay (Argentina)
}

\author{
Cowper Coles, F.; Sánchez, S.; Jorge, L.C. \\ Instituto de Ictiología, Facultad de Ciencias Veterinarias, Universidad Nacional del Nordeste, \\ Sargento Cabral 2139, Corrientes (3400), Argentina. Tel/fax 03783-425753. \\ E-mail: francisco_cowpercoles@hotmail.com.
}

\begin{abstract}
Resumen
Cowper Coles, F.; Sánchez, S.; Jorge, L.C.: Estructura cariotípica de dos poblaciones de Rhamdia quelen (Pisces, Siluriformes) de los ríos Paraná y Paraguay (Argentina). Rev. vet. 24: 2, 144-147, 2013. Se analizaron 13 ejemplares de Rhamdia quelen (4 hembras y 9 machos) capturados en el río Paraná y 7 ejemplares (3 hembras y 4 machos) del río Paraguay. En total se registraron 244 metafases. Los especimenes mostraron un número diploide de 58 cromosomas. Con una formula cariotípica de $46 \mathrm{M} / \mathrm{SM}+12 \mathrm{ST}$, el número fundamental (FN) fue de 116. No se registraron diferencias entre ambas poblaciones. No se evidenció la ocurrencia de heteromorfismo cromosómico relacionado con los sexos. En ambas poblaciones, las regiones organizadoras de nucléolos (NOR) se localizaron en la región terminal del brazo corto de un par subtelocéntrico (par 27). Los cromosomas del complemento A mostraron bandas $\mathrm{C}+$ distribuidas principalmente sobre las NOR, en las regiones terminales y pericentroméricas de unos pocos cromosomas. Se concluye que las poblaciones de $R$. quelen analizadas mantuvieron su macro-estructura cariotípica conservada con respecto al número diploide de 58 , con cromosomas de dos brazos, región organizadora de nucléolos de tipo simple y un patrón pobre de bandas $\mathrm{C}$.
\end{abstract}

Palabras clave: Rhamdia quelen, cariotipo, bandeo-C, NOR, ríos Paraná y Paraguay.

\begin{abstract}
Cowper Coles, F., Sánchez, S.; Jorge, L.C.: Cytogenetic studies of two populations of Rhamdia quelen (Pisces, Siluriformes) from the Paraná and Paraguay rivers (Argentina). Rev. vet. 24: 2, 144-147, 2013. Thirteen specimens of Rhamdia quelen captured in the Paraná river ( 4 females and 9 males) and 7 specimens from the Paraguay river ( 3 females and 4 males), were studied. In total, 244 metaphases were registered. $R$. quelen showed a diploid number of 58 chromosomes. With a karyotype formula of $46 \mathrm{M} / \mathrm{SM}+12 \mathrm{ST}$, the fundamental number (FN) was 116; there were no differences between the two populations. There was no evidence of occurrence of chromosomal heteromorphism related to sex. The nucleolus organizer regions (NOR) in the two populations were located in the terminal region of the short arm of a subtelocentric pair (pair 27). The A complement chromosomes showed bands $\mathrm{C}+$ distributed mainly above the NOR on pericentromeric and terminal regions in a few chromosomes. It is concluded that for the analyzed populations of $R$. quelen, the karyotypic features are conserved regarding the diploid number of 58, with two-arms chromosomes, single nucleolar organizer region, and poor $\mathrm{C}$ banding pattern.
\end{abstract}

Key words: Rhamdia quelen, caryotype, C-banding, NOR, Paraná and Paraguay rivers.

\section{INTRODUCCIÓN}

Rhamdia quelen es una especie que pertenece al orden Siluriformes. Posee una amplia distribución en Argentina, que incluye las cuencas de los ríos Paraná, Paraguay, Uruguay y Río de la Plata. En la actualidad $R$. quelen es un sinónimo de al menos 47 especies de este género, su situación taxonómica sigue siendo controvertida ${ }^{3,8}$.

Recibido: 19 octubre 2012 / Aceptado: 22 febrero 2013
Desde el punto de vista citogenético $R$. quelen exhibe un número diploide de 58 cromosomas. Sin embargo, estudios realizados en diferentes poblaciones reveló que la especie presenta discrepancias en las fórmulas cariotípicas 4, 5, 8, 10, 12, 18, 19, 22 . En la especie también se evidenció la presencia de cromosomas $\mathrm{B}$, responsables de la variación intra e interindividual en el número cromosómico ${ }^{5,8,10,12,18,19,22}$. Tales aspectos citogenéticos, combinados con los problemas taxonómicos, hacen posible que $R$. quelen sea considerada como un "complejo de especies". 
En la actualidad la especie es de interés en piscicultura por presentar aptitudes muy convenientes como ser su rápido crecimiento, fácil adaptación a la cría intensiva, rusticidad, reproducción artificial en laboratorios, alta tasa de fecundación, tolerancia a un amplio rango de temperaturas, buen peso final, carne sabrosa con bajo tenor de gordura y pocas espinas ${ }^{2,17}$.

Por su diversidad cariotípica e interés económico en piscicultura, el objetivo del presente trabajo fue investigar la estructura cariotípica de ejemplares de $R$. quelen provenientes de diferentes sitios de colecta de los ríos Paraná y Paraguay.

\section{MATERIAL Y MÉTODOS}

La captura de los peces se realizó sobre las cuencas de los ríos Paraná (en las localidades de Ita Ibaté y Ya- hapé) y Paraguay (en la ciudad de Formosa). Los ejemplares fueron transportados al laboratorio en bolsas de polietileno que contenían agua y oxígeno, siendo luego mantenidos en peceras debidamente acondicionadas.

Se analizaron 23 especimenes de $R$. quelen, obteniéndose resultados satisfactorios en 13 individuos (4 hembras y 9 machos) del río Paraná y 7 ejemplares (3 hembras y 4 machos) del río Paraguay. En total se registraron 244 metafases. Previo al sacrificio los animales fueron anestesiados con metasulfonato de tricaina MS222 (Finquel $\AA^{\circledR}$ ).

Para el estudio de los cromosomas se utilizó la porción anterior del riñón ${ }^{21}$. Los cromosomas mitóticos se obtuvieron a través de la disociación del órgano en cubetas que contenían $10 \mathrm{ml}$ de solución de Hank; posteriormente se continúo con los procedimientos de rutina ${ }^{6}$. Las regiones organizadoras de nucleolos (NOR)

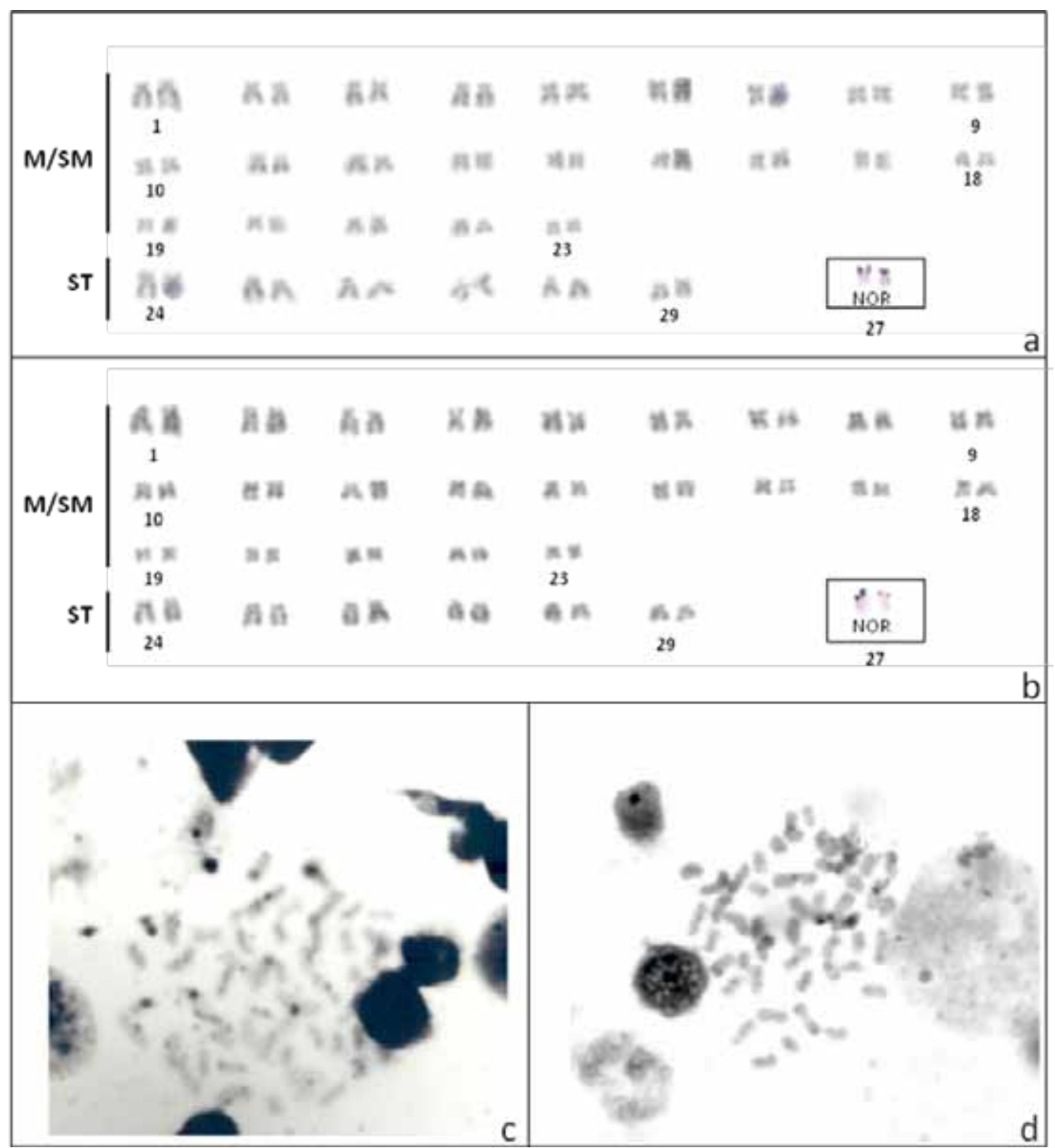

Figura 1. (a) cariotipo de Rhamdia quelen proveniente del río Paraná (Ita Ibaté); (b) cariotipo de R. quelen del río Paraguay (Formosa); (a y b) en los recuadros se observan los cromosomas portadores de la NOR luego de la coloración con nitrato de plata; (c y d) metafases con bandeo $\mathrm{C}$ de ejemplares provenientes de los ríos Paraná (c) y Paraguay (d). 
se identificaron por tinción con nitrato de plata ${ }^{13}$. La heterocromatina constitutiva se localizó por el método convencional de bandeo $\mathrm{C}^{23}$.

Las metafases fueron fotografiadas con el auxilio del kit digital Infinity 1 acoplado a un microscopio óptico Olympus BX41, posteriormente fueron analizadas con el programa analizador de imágenes Image ProPlus 5.1. En el cariotipo se distribuyeron los pares homólogos en orden decreciente de tamaño. Los cromosomas se clasificaron como metacéntrico-submetacéntrico (M/SM) y subtelocéntrico (ST) de acuerdo a su morfología y relación de brazos ${ }^{16}$.

\section{RESULTADOS Y DISCUSIÓN}

En ambas poblaciones analizadas (ríos Paraná y Paraguay), los especimenes de $R$. quelen presentaron un número diploide de 58 cromosomas. Las fórmulas cariotípicas de los ejemplares de estas poblaciones fue de $46 \mathrm{M} / \mathrm{SM}+12 \mathrm{ST}$, el número fundamental (FN) fue de 116 (Figura 1, a y b).

$R$. quelen presentó $2 \mathrm{n}=58$ cromosomas en todas las poblaciones estudiadas hasta el momento. El mantenimiento de este número diploide puede ser considerado como una característica de la especie. Si bien $R$. quelen conserva el numero diploide, no ocurre lo mismo con la composición cromosómica, en la que se observa una acentuada variabilidad con predominio de cromosomas bi-armados 4, 5, 8, 10, 12, 18, 19, 22. Variaciones envolviendo la fórmula cariotípica, con mantenimiento del número diploide, indican que reordenamientos norobertsonianos desempeñan un importante papel en la evolución de los cromosomas. Inversiones cromosómicas (peri- o paracéntricas) han sido consideradas como los principales mecanismos de los cambios cariotípicos dentro de algunos grupos Siluriformes ${ }^{8}$.

En las poblaciones estudiadas no se observaron cromosomas B. La ausencia de estos cromosomas no es una condición muy común en el género Rhamdia, si bien existen relatos de no haber sido encontrados en algunas poblaciones de $R$. quelen ${ }^{4,18,22}$. En el presente estudio no se observó heteromorfismo cromosómico relacionado con los sexos.

En ambas poblaciones las NOR se encontraron en un par de cromosomas subtelocéntricos (par 27), en posición terminal del brazo corto, coincidentes con las constricciones secundarias (Figura 1, a y b). En el género Rhamdia, la ubicación y el número de NOR es constante entre las diferentes especies y poblaciones estudiadas, existiendo un par de NOR situado en los brazos cortos de cromosomas submeta- o subtelocéntrico $5,7,8$, 19, 22 . Como excepción se citan casos de Rhamdia branneri y Rhamdia sp que mostraron un sistema de NOR múltiple, hallazgo considerado como un carácter apomórfico en el género ${ }^{1}$.

Ocasionalmente se observó una variación en el tamaño de las regiones nucleolares, donde uno de los cromosomas presentó una marcación mayor en relación a su homólogo. Este heteromorfismo de tamaño podría haberse debido a un polimorfismo estructural de los cistrones ribosómicos y no a un patrón diferencial de expresión de las NOR como fue comprobado en otras especies ${ }^{15}$. El tamaño polimórfico de las NOR entre los cromosomas homólogos podría ser explicado por eventos espontáneos de duplicación, delección y crossing-over desigual ${ }^{9}$. El heteromorfismo en el tamaño de las NOR ha sido frecuentemente reportado en peces neotropicales ${ }^{11,14,20,24}$, entre otros. El bandeo $\mathrm{C}$ reveló pequeña cantidad de heterocromatina constitutiva distribuida principalmente sobre la NOR, en las regiones terminales y pericentromérica de unos pocos cromosomas (Figura 1, c y d). Este patrón de heterocromatina es el más común encontrado en los cromosomas del complemento en los especimenes de $R$. quelen analizados hasta el momento 5, 8, 10, 18, 19,22.

Los datos obtenidos de las dos poblaciones analizadas de $R$. quelen permiten concluir que ellas mantienen su macro-estructura cariotípica conservada con respecto al número diploide de 58 cromosomas, con dos brazos, región organizadora de nucléolos de tipo simple y un patrón pobre de bandas $\mathrm{C}$.

\section{REFERENCIAS}

1. Abucarma M, Martins-Santos IC. 2001 Karyotype and B chromosome of Rhamdia species (Pisces, Pimelodidae) endemic in the River Iguaçu basin. Cytologia 66: 299-306.

2. Baldisserotto B, Radünz Neto J. 2004. Criação de jundiá, Ed. UFSM, Santa Maria, Brasil, 232 p.

3. Casciotta J, Almirón A, Bechara J. 2005. Peces del Iberá. Hábitat y diversidad, Ed. Graficar, La Plata, Argentina, $244 \mathrm{p}$.

4. Fenocchio AS, Bertollo LA, Takahashi CS, Dias AL, Swarça AC. 2003. Cytogenetic studies and correlate considerations on Rhamdiinae relationships (Pisces, Siluroidei, Pimelodidae). Cytologia 68: 363-368.

5. Fenocchio AS, Swarça AC, Cestari MM, Dias AL. 2003. Karyotypic characterization and NOR analysis by different banding techniques of Rhamdia quelen (Pisces, Pimelodidae) from the first plateau of the Iguaçu River (Brazil). Folia Biol 51: 219-222.

6. Foresti F, Oliveira C, Almeida Toledo LF. 1993. A method for chromosome preparations from large fish specimens using in vitro short-term treatment with colchicine. Experientia 49: 810-813.

7. Garcia C, Moreira-Filho O, Bertollo LA, Centofante L. 2003. B chromosomes and natural triploidy in Rhamdia sp. (Pisces, Siluriformes, Heptapteridae). Cytologia 68: 403-411.

8. Garcia C, Oliveira, C, Almeida-Toledo LF. 2010. Karyotypic evolution trends in Rhamdia quelen (Siluriformes, Heptapteridae) with considerations about the origin and differentiation of its supernumerary chromosomes. Gen \& Molec Res 9: 365-384.

9. Gold JR, Li C, Shipley NS, Powers PK. 1990. Improved methods for working with chromosomes with a review of metaphase chromosome banding. J Fish Biol 37: 563-575. 
10. Guilherme LC. 2005. Estudos reprodutivos e citogenéticos na população de Rhamdia quelen (Pisces, Rhamdiidae) do rio Uberabinha no município de Uberlândia - MG e desenvolvimento de sistema artesanal de recirculação d'água para criação de peixes. $P h D$ Thesis, Universidade Federal de Uberlândia, Brazil, 85 p.

11. Hatanaka T, Galetti PM. 2004. Mapping of the 18 S and 5S ribosomal RNA genes in the fish Prochilodus argenteus Agassiz 1829 (Characiformes, Prochilodontidae). Genetica 122: 239-244.

12. Hochberg VB, Erdtmann B. 1988. Cytogenetical and morphological considerations on Rhamdia quelen (Pisces, Pimelodidae). The occurrence of B chromosomes and polymorphic NOR regions. Brazil J Genetics 11: 563-576.

13. Howell WM, Black DA. 1980. Controlled silver-staining of nucleolus organizer regions with a protective colloidal developer: a 1-step method. Experientia 36: 1014-1015.

14. Jesus CM, Moreira-Filho O. 2000. Cytogenetics studies in some Apareiodon species (Pisces, Parodontidae). Cytologia 65: 397-402.

15. Jesus CM, Moreira-Filho O. 2003. Chromosomal location of 5S and 18S rRNA genes in Prochilodus lineatus (Characiformes, Prochilodontidae). Caryologia 56: 281-287.

16. Levan A, Fredga K, Sandberg AA. 1964. Nomenclature for centromeric position on chromosomes. Hereditas 52: 201-220.

17. Luchini L, Averdano T. 1985. Primeros resultados de cultivo de un pez de aguas cálidas (Rhamdia sapo) con fines de producción y consumo humano. Rev Arg Prod Anim 4: 621-629.
18. Martínez JF, Lui RL, Blanco DR, Traldi JB, Silva LF, Venere PC, Souza IL, Moreira-Filho O. 2011. Comparative cytogenetics of three populations from the Rhamdia quelen species complex (Siluriformes, Heptapteridae) in two Brazilian hydrographic basins. Cytologia 64: 121-128.

19. Moraes VP, Cereali SS, Froehlich O, Dias AL. 2007. Cytogenetic characterization of Rhamdia quelen (Siluriformes, Heptapteridae) from the Bodoquena Plateau, Mato Grosso do Sul, Brasil) Genet \& Molec Res 6: 627-633.

20. Moreira-Filho O, Bertollo LA, Galetti PM. 1984. Structure and variability of nucleolar organizer regions in Parodontidae fish. Can J Genet Cytology 26: 564-568.

21. Moreira-Filho O, Bertollo LA. 1991. Extraction and use of the cephalic kidney for chromosome studies in small fish. Brazil J Genet 14: 1085-1090.

22. Stivari MK, Martins-Santos IC. 2004. Karyotipe diversity in two populations of Rhamdia quelen (Pisces, Heptapteridae). Cytologia 69: 25-34.

23. Sumner AT. 1972. A simple technique for demonstrating centromeric heterocromation. Exp Cell Res 75: 304-306.

24. Wasko AP, Galetti M. 2000. Mapping $18 \mathrm{~S}$ ribosomal genes in fish of the genus Brycon (Characidae) by fluorescent in situ hybridization (FISH). Genet Mol Biol 23: 135-138. 